\title{
On the Origins of Iterative Substructuring Methods
}

\author{
Martin J. Gander and Xuemin Tu
}

\section{The Invention of Substructuring Methods}

Substructuring methods were invented in the engineering community. A very early precursor is the so called "Moment Distribution Method", or "Hardy Cross Method" named after its inventor [11]. Cross states in the introduction to his paper from 1930 his motivation for the method:

The reactions in beams, bents, and arches which are immovably fixed at their ends have been extensively discussed. They can be found comparatively readily by methods which are more or less standard. The method of analysis herein presented enables one to derive from these the moments, shears, and thrusts required in the design of complicated continuous frames.

The idea is to give a precise method how to combine structures for which their reaction to load is known (i.e. tabulated), when they interact at joints between structures. The method is iterative, and described in Figure 1.

In modern terms, it is a Jacobi relaxation applied to the displacement formulation of structural analysis [39], but also a precursor to the finite element method.

$\therefore$ The method of moment distribution is this: (a) Imagine all joints in the structure held so that they cannot rotate and compute the moments at the ends of the members for this condition; $(b)$ at each joint distribute the unbalanced fixed-end moment among the connecting members in proportion to the constant for each member defined as "stiffness"; (c) multiply the moment distributed to each member at a joint by the carry-over factor at that end of the member and set this product at the other end of the member; $(d)$ distribute these moments just "carried over"; $(e)$ repeat the process until the moments to be carried over are small enough to be neglected; and $(f)$ add all moments -fixed-end moments, distributed moments, moments carried over-at each end of each member to obtain the true moment at the end.

To the mathematically inclined the method will appear as one of solving a series of normal simultaneous equations by successive approximation. From an engineering viewpoint it seems simpler and more useful to think of the solution as if it were a physical occurrence.

Fig. 1 The Hardy Cross Method from 1930

Section de Mathématiques, Université de Genève, CP 64, 1211 Genève, Switzerland Martin.Gander@unige.ch · Department of Mathematics, University of Kansas, 1460 Jayhawk Blvd, Lawrence, KS 66045-7594, U.S.A. xtu@math.ku.edu 

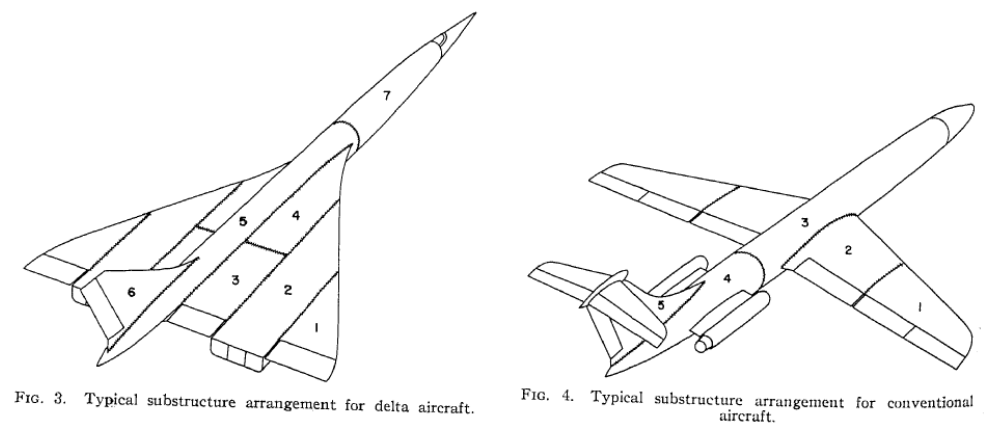

Fig. 2 Two plane structures with non-overlapping subdomain decompositions from the original publication of Przemieniecki in 1963

It was however at Boeing, right after the reinvention of the finite element method for the design of aircraft $[38,9]$, where Przemieniecki introduced in his seminal paper [33] the first substructuring method of the form we know them now. He first explains why substructuring became necessary:

The necessity for dividing a structure into substructures arises either from the requirement that different types of analysis have to be used on different components, or because the capacity of the digital computer is not adequate to cope with the analysis of the complete structure.

At the time, computational techniques for the simulation of aircraft were rapidly developing, and complex structures had to be simulated, as shown in the original drawings of Przemieniecki in Figure 2. Unlike in the case of Cross, the substructures were too complicated to have tabulated solutions, and had to be simulated as well. At the beginning of his paper, Przemieniecki describes the idea of his domain domain decomposition method, which is not so different from the method of Cross, but it is not iterative:

In the present method each substructure is first analyzed separately, assuming that all common boundaries with adjacent substructures are completely fixed: these boundaries are then relaxed simultaneously and the actual boundary displacements are determined from the equations of equilibrium of forces at the boundary joints. The substructures are then analyzed separately again under the action of specified external loading and the previously determined boundary displacements.

Let us see how this can be written in mathematical terms, using the notation used by Przemieniecki. Like for many structural engineers at that time, the reasoning was at the discrete level: let $P$ be the exterior forces, $K$ the stiffness matrix, and $U$ the displacement vector. Then these quantities satisfy the system of equations

$$
K U=P .
$$

We now partition the unknowns $U$ into unknowns $U_{i}$ in the interior of each substructure, and the unknowns $U_{b}$ on the boundaries between substructures, as indicated in 
Figure 2. If we partition the matrix and right hand side accordingly, the system (1) can be rewritten as

$$
\left[\begin{array}{cc}
K_{b b} & K_{b i} \\
K_{i b} & K_{i i}
\end{array}\right]\left[\begin{array}{c}
U_{b} \\
U_{i}
\end{array}\right]=\left[\begin{array}{c}
P_{b} \\
P_{i}
\end{array}\right]
$$

Now the algorithm of Przemieniecki has three steps, as we have seen above. The first step must keep boundaries between substructures fixed, and hence an (unknown) force $P^{(\alpha)}$ is needed to keep these boundaries fixed. Przemieniecki therefore partitions the forcing vector into

$$
P=P^{(\alpha)}+P^{(\beta)}=\left[\begin{array}{c}
P_{b}^{(\alpha)} \\
P_{i}
\end{array}\right]+\left[\begin{array}{c}
P_{b}^{(\beta)} \\
0
\end{array}\right] .
$$

Since with the first vector on the right hand side as a load, the boundaries of the substructures do not move, the displacements can also be written in the same decomposition, namely

$$
U=U^{(\alpha)}+U^{(\beta)}=\left[\begin{array}{c}
0 \\
U_{i}^{(\alpha)}
\end{array}\right]+\left[\begin{array}{c}
U_{b} \\
U_{i}^{(\beta)}
\end{array}\right] .
$$

By linearity, we can rewrite the original system as two systems, which represent the first two steps in Przemieniecki's algorithm,

$$
(\alpha):\left[\begin{array}{cc}
K_{b b} & K_{b i} \\
K_{i b} & K_{i i}
\end{array}\right]\left[\begin{array}{c}
0 \\
U_{i}^{(\alpha)}
\end{array}\right]=\left[\begin{array}{c}
P_{b}^{(\alpha)} \\
P_{i}
\end{array}\right]
$$

and

$$
(\beta):\left[\begin{array}{cc}
K_{b b} & K_{b i} \\
K_{i b} & K_{i i}
\end{array}\right]\left[\begin{array}{c}
U_{b} \\
U_{i}^{(\beta)}
\end{array}\right]=\left[\begin{array}{c}
P_{b}^{(\beta)} \\
0
\end{array}\right] .
$$

In the first step of Przemieniecki's algorithm one needs to solve the first system. Because the interfaces between substructures are not moving, this system simplifies to

$$
K_{b i} U_{i}^{(\alpha)}=P_{b}^{(\alpha)}, \quad K_{i i} U_{i}^{(\alpha)}=P_{i} .
$$

Knowing the forces $P_{i}$ in the interior of each substructure, we can compute the interior displacements when the interfaces are kept fixed, $U_{i}^{(\alpha)}=K_{i i}^{-1} P_{i}$. Inserting this result into the first equation uncovers the unknown force that Przemieniecki needed to impose to keep the interfaces fixed, namely

$$
P_{b}^{(\alpha)}=K_{b i} K_{i i}^{-1} P_{i}
$$

We can now determine the remaining forces $P_{b}^{(\beta)}$ on the interfaces,

$$
P_{b}^{(\beta)}=P_{b}-P_{b}^{(\alpha)}=P_{b}-K_{b i} K_{i i}^{-1} P_{i},
$$

and inserting this result into the second system $(\beta)$ gives 


$$
K_{b b} U_{b}+K_{b i} U_{i}^{(\beta)}=P_{b}^{(\beta)}, \quad K_{i b} U_{b}+K_{i i} U_{i}^{(\beta)}=0 .
$$

We can now compute the second step in Przemieniecki's algorithm, namely the response of the structures to the interface loading $P_{b}^{(\beta)}$. The second equation gives the internal displacement $U_{i}^{(\beta)}$ based on the boundary displacement $U_{b}$,

$$
U_{i}^{(\beta)}=-K_{i i}^{-1} K_{i b} U_{b},
$$

and inserting this into the first equation, Przemieniecki obtains for the unknowns at the interfaces the system

$$
\left(K_{b b}-K_{b i} K_{i i}^{-1} K_{i b}\right) U_{b}=P_{b}-K_{b i} K_{i i}^{-1} P_{i}
$$

We see that the procedure, which Przemieniecki motivated by a strictly mechanical argument, leads simply to the Schur complement system, where all interior variables are eliminated! We note that the Schur complement system can also be derived using discrete harmonic functions on the substructures. The third and last step, after solving the Schur complement system, is to simply compute the corresponding interior displacements, and the problem is solved. Historically, the Schur complement was also known under the name capacitance matrix [25], as we will see next.

\section{Capacitance Matrix Methods}

The capacitance matrix method became popular in the early 1970, due to a publication by Buzbee, Dorr, George and Golub [8] that has a very short abstract:

There are several very fast direct methods which can be used to solve the discrete Poisson equation on rectangular domains. We show that these methods can also be used to treat problems on irregular regions.

The paper first gives a general introduction to Schur complement techniques at the algebraic level, and then the authors show how Schur complements can be used to solve problems on irregular domains by imbedding, and by domain splitting, with a typical example of an L-shaped domain. As in Przemieniecki, the Schur complement system (5) is solved by direct methods. A new important idea was then introduced by Proskurowski and Widlund in [32]:

This new formulation leads to well-conditioned capacitance matrix equations which can be solved quite efficiently by the conjugate gradient method. A highly accurate solution can, therefore, be obtained at an expense which grows no faster than that for a fast Laplace solver on a rectangle when the mesh size is decreased.

The authors explain that their method can use fast Possion solvers for a similar purpose as for the fundamental solustions when constructing the classical integral equations of potential theory. The key contribution is however the solution of the Schur complement system by a Krylov method, which paved the way for iterative substructuring methods. 


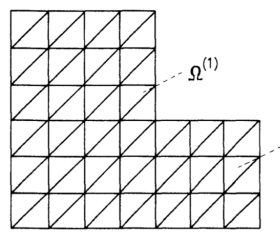

$$
\begin{aligned}
& \gamma_{0}\left(K^{1 / 2} y, y\right)_{R^{p}} \leqq(C y, y)_{R^{p}} \leqq \gamma_{1}\left(K^{1 / 2} y, y\right)_{R^{p}} \\
& \Omega_{0} \text { and } \gamma_{1} \text { are the following constants } \\
& \Omega_{0}=r /\left(1+\left(1+r^{2}\right)^{1 / 2}\right), \quad \gamma_{1}=(1+2 r(1+2 \sigma)) b_{2} /\left(4 r b_{1}\right) \\
& r=(32)^{1 / 2}\left(a_{2}-a_{1}\right) / b_{1} .
\end{aligned}
$$

Fig. 3 Original Figure by Dryja [16] to introduce preconditioned iterative substructuring methods on the left, and first preconditioner estimate on the right

\section{Iterative Substructuring Methods}

The explicit calculation of the Schur complement $S$ is expensive and requires large amount of memory since the matrix is much denser than the original stiffness matrix $K$ as defined in (1), even though it is much smaller. However the action of the Schur complement on a vector can be calculated implicitly by solving local substructure problems. Therefore the explicit formation of the Schur complement can be avoided if Krylov space methods are used to solve the interface problem (5) iteratively, as shown in [32]. To make the number of iterations however manageable, for certain accuracy, it is crucial to construct a suitable preconditioner for the Krylov subspace methods. In a sequence of papers $[15,16,17]$, Dryja first introduced preconditioned Krylov space methods for solving the interface problem (5). The L-shaped domain shown in Figure 3 on the left was divided into two subdomains in [16], and the preconditioner is selected as $K^{-1 / 2}$, where $K$ is here the discrete Laplacian operator on the subdomain interface. Dryja proved in [16] the first spectral equivalence result for preconditioning the capacitance matrix, as shown in Figure 3 on the right. This result was proved using Fourier analysis, and the preconditioner can also be implemented efficiently using a fast sine transform.

Golub and Mayers proposed a slightly improved version of this preconditioners in [24]. In [1, 2], Bjørstad and Widlund explicit derived and diagonalized the local Schur complement $S^{(i)}$ and proposed two preconditioners. The preconditioner considered by Dryja was called the "good method" and the other, the NeumannDirichlet preconditioner for two subdomains, the "excellent method". The application of this preconditioner to a vector requires the solution of one subdomain Neumann problem and one subdomain Dirichlet problem. It converges in one step if the two subdomains come from a symmetric region cut in half and the triangulation is regular and symmetric. If the subdomain partition allows a red-black coloring, the Neumann-Dirichlet (Dirichlet-Neumann) algorithms can also be extended to many subdomains.

Another type of preconditioner, the Neumann-Neumann preconditioner was introduced in $[22,3,27]$. The application of this preconditioner to a vector requires the solutions of two Dirichlet problems and two Neumann problems. Thus, it is more expensive than the Neumann-Dirichlet preconditioner. However, it is easy to extend to many subdomains and can be made to perform well with jump coefficients by introducing a simple scaling operator. 
The number of iterations will increase with an increase of the number of subdomains for most one-level preconditioners. An additional level is needed to remove such dependence. For Dirichlet-Neumann preconditioners, when the subdomain partitions has cross points, a natural second, coarse level solver can be formed using variables related to these cross points, see [19, 18]. The two-level NeumannNeumann algorithms, known as Balancing Neumann-Neumann algorithms, where introduced in [29, 28, 26, 21]. The coarse level solver can be constructed using weighted counting functions. The balancing Neumann-Neumann algorithm has been extended to several applications such as for the mixed finite element discretizations, Stokes, and almost incompressible elasticity, [10, 31, 23]. Recently, the balancing domain decomposition by constraints method has been developed and it has been widely used $[12,30]$; it is similar to the balancing Neumann-Neumann algorithms but its coarse problems are given in terms of a set of primal constraints partially enforcing contiunity across the interface.

\section{Primal Iterative Substructuring Methods}

There is another class of substructuring methods known as the primal iterative substructuring methods. The difference between the preconditioners in this class and the algorithms described in Section 3 is that the coupling between all pairs of faces, edges, and vertices are eliminated in the preconditioners of this class while the coupling between neighboring subdomains are eliminated in the previous class.

The development of the primal iterative substructuring methods started with a famous series of four papers $[4,5,6,7]$. [4] is the first paper on iterative substructuring methods to deal with cross points satisfactorily. The algorithm proposed in that paper has a coarse level component formed in terms of the cross points and an almost optimal condition number bound was established in two dimensions. However such a coarse level problem does not always work well in three dimensions because of a much weaker finite element Sobolev inequality. Related methods with a coarse solver based on the wire basket were introduced in [7] for three dimensional problems and an almost optimal bound was obtained.

An observation on using a change of basis from a partial hierarchical basis to the usual nodal basis for this class of algorithms was made in [37] and many preconditioners of this type were introduced in [20]. These algorithms have been successfully implemented and extended to three dimensional linear elasticity [35, 36]. Quite recently, the coarse components introduced here have also been used for overlapping domain decomposition methods to obtain algorithms independent of the coefficient jumps $[34,13,14]$.

Acknowledgements This work was supported in part by National Science Foundation Contract No. DMS-1115759. 


\section{References}

1. Petter E. Bjørstad and Olof B. Widlund. Solving elliptic problems on regions partitioned into substructures. In Garrett Birkhoff and Arthur Schoenstadt, editors, Elliptic Problem Solvers II, pages 245-256, New York, 1984. Academic Press.

2. Petter E. Bjørstad and Olof B. Widlund. Iterative methods for the solution of elliptic problems on regions partitioned into substructures. SIAM J. Numer. Anal., 23(6):1093-1120, 1986.

3. Jean-François Bourgat, Roland Glowinski, Patrick Le Tallec, and Marina Vidrascu. Variational formulation and algorithm for trace operator in domain decomposition calculations. In Tony Chan, Roland Glowinski, Jacques Périaux, and Olof Widlund, editors, Domain Decomposition Methods. Second International Symposium on Domain Decomposition Methods, pages 3-16, Philadelphia, PA, 1989. SIAM. Los Angeles, California, January 14-16, 1988.

4. James H. Bramble, Joseph E. Pasciak, and Alfred H. Schatz. The construction of preconditioners for elliptic problems by substructuring, I. Math. Comp., 47(175):103-134, 1986.

5. James H. Bramble, Joseph E. Pasciak, and Alfred H. Schatz. The construction of preconditioners for elliptic problems by substructuring, II. Math. Comp., 49(179):1-16, 1987.

6. James H. Bramble, Joseph E. Pasciak, and Alfred H. Schatz. The construction of preconditioners for elliptic problems by substructuring, III. Math. Comp., 51(184):415-430, 1988.

7. James H. Bramble, Joseph E. Pasciak, and Alfred H. Schatz. The construction of preconditioners for elliptic problems by substructuring, IV. Math. Comp., 53(187):1-24, 1989.

8. B.L Buzbee, F.W. Dorr, J.A. George, and G.H. Golub. The direct solution of the discrete Poisson equation on irregular regions. SIAM J. Numer. Anal., 8(4):722-736, 1971.

9. Ray W. Clough. The finite element method in plane stress analysis. In Proc ASCE Conf Electron Computat, Pittsburg, PA, 1960.

10. Lawrence C. Cowsar, Jan Mandel, and Mary F. Wheeler. Balancing domain decomposition for mixed finite elements. Math. Comp., 64(211):989-1015, July 1995.

11. Hardy Cross. Analysis of continuous frames by distributing fixed-end moments. Transactions of the American Society of Civil Engineers, (Paper 1793):1-10, 1930.

12. Clark R. Dohrmann. A preconditioner for substructuring based on constrained energy minimization. SIAM J. Sci. Comput., 25(1):246-258, 2003.

13. Clark R. Dohrmann and Olof B. Widlund. An overlapping Schwarz algorithm for almost incompressible elasticity. SIAM J. Numer. Anal., 47(4):2897-2923, 2009.

14. Clark R. Dohrmann and Olof B. Widlund. Hybrid domain decomposition algorithms for compressible and almost incompressible elasticity. Internat. J. Numer. Methods Engrg., 82(2):157183,2010

15. Maksymilian Dryja. An algorithm with a capacitance matrix for a variational-difference scheme. In Guri I. Marchuk, editor, Variational-Difference Methods in Mathematical Physics, pages 63-73, Novosibirsk, 1981. USSR Academy of Sciences.

16. Maksymilian Dryja. A capacitance matrix method for Dirichlet problem on polygon region. Numer. Math., 39:51-64, 1982.

17. Maksymilian Dryja. A finite element-capacitance method for elliptic problems on regions partitioned into subregions. Numer. Math., 44:153-168, 1984.

18. Maksymilian Dryja. A method of domain decomposition for 3-D finite element problems. In Roland Glowinski, Gene H. Golub, Gérard A. Meurant, and Jacques Périaux, editors, First International Symposium on Domain Decomposition Methods for Partial Differential Equations, pages 43-61, Philadelphia, PA, 1988. SIAM. Paris, France, January 7-9, 1987.

19. Maksymilian Dryja, Wlodek Proskurowski, and Olof Widlund. A method of domain decomposition with crosspoints for elliptic finite element problems. In Blagovest Sendov, editor, Optimal Algorithms, pages 97-111, Sofia, Bulgaria, 1986. Bulgarian Academy of Sciences.

20. Maksymilian Dryja, Barry F. Smith, and Olof B. Widlund. Schwarz analysis of iterative substructuring algorithms for elliptic problems in three dimensions. SIAM J. Numer. Anal., 31(6):1662-1694, December 1994.

21. Maksymilian Dryja and Olof B. Widlund. Schwarz methods of Neumann-Neumann type for three-dimensional elliptic finite element problems. Comm. Pure Appl. Math., 48(2):121-155, February 1995. 
22. Roland Glowinski and Mary F. Wheeler. Domain decomposition and mixed finite element methods for elliptic problems. In Roland Glowinski, Gene H. Golub, Gérard A. Meurant, and Jacques Périaux, editors, First International Symposium on Domain Decomposition Methods for Partial Differential Equations, pages 144-172, Philadelphia, PA, 1988. SIAM. Paris, France, January 7-9, 1987.

23. Paulo Goldfeld, Luca F. Pavarino, and Olof B. Widlund. Balancing Neumann-Neumann preconditioners for mixed approximations of heterogeneous problems in linear elasticity. Numer. Math., 95(2):283-324, 2003.

24. Gene Golub and D. Mayers. The use of preconditioning over irregular regions. In R. Glowinski and Jacques-Louis. Lions, editors, Computing Methods in Applied Sciences and Engineering, VI, pages 3-14, Amsterdam, New York, Oxford, 1984. North-Holland. Proceedings of a conference held in Versailles, France, December 12-16,1983.

25. R.W. Hockney. Potential calculation and some application. Methods Comput. Phys., 9:135211, 1970.

26. Patrick Le Tallec. Domain decomposition methods in computational mechanics. In J. Tinsley Oden, editor, Computational Mechanics Advances, volume 1 (2), pages 121-220. NorthHolland, 1994.

27. Patrick Le Tallec, Yann-Hervé De Roeck, and Marina Vidrascu. Domain decomposition methods for large linearly elliptic three-dimensional problems. J. Comput. Appl. Math., 34(1):93$117,1991$.

28. Jan Mandel. Balancing domain decomposition. Comm. Numer. Meth. Engrg., 9:233-241, 1993.

29. Jan Mandel and Marian Brezina. Balancing domain decomposition: Theory and computations in two and three dimensions. Technical Report UCD/CCM 2, Center for Computational Mathematics, University of Colorado at Denver, 1993.

30. Jan Mandel and Clark R. Dohrmann. Convergence of a balancing domain decomposition by constraints and energy minimization. Numer. Linear Algebra Appl., 10(7):639-659, 2003.

31. Luca F. Pavarino and Olof B. Widlund. Balancing Neumann-Neumann methods for incompressible Stokes equations. Comm. Pure Appl. Math., 55(3):302-335, March 2002.

32. W. Proskurowski and O. Widlund. On the numerical solution of helmholtz's equation by the capacitance matrix method. Math. of Comp., 30(135):433-468, 1976.

33. J. S. Przemieniecki. Matrix structural analysis of substructures. Am. Inst. Aero. Astro. J., $1: 138-147,1963$.

34. Marcus V. Sarkis. Nonstandard coarse spaces and Schwarz methods for elliptic problems with discontinuous coefficients using non-conforming elements. Numer. Math., 77(3):383-406, 1997.

35. Barry F. Smith. Domain Decomposition Algorithms for the Partial Differential Equations of Linear Elasticity. PhD thesis, Courant Institute of Mathematical Sciences, September 1990. Tech. Rep. 517, Department of Computer Science, Courant Institute.

36. Barry F. Smith. An optimal domain decomposition preconditioner for the finite element solution of linear elasticity problems. SIAM J. Sci. Statist. Comput., 13(1):364-378, January 1992.

37. Barry F. Smith and Olof B. Widlund. A domain decomposition algorithm using a hierarchical basis. SIAM J. Sci. Stat. Comput., 11(6):1212-1220, 1990.

38. N. J. Turner, R. W. Clough, H. C. Martin, and L. J. Topp. Stiffness and deflection analysis of complex structures. J. Aero. Sci., 23:805-23, 1956.

39. K. Y. Volokh. On foundation of the Hardy Cross method. International Journal of Solids and Structures, 39(16):4197-4200, 2002. 